\title{
Transurethral En Bloc Resection of an Urachal Cyst
}

\author{
Toru Etsunaga, ${ }^{1}$ Yoshihiro Ono, ${ }^{1} \quad$ Tomoko Koya, ${ }^{1}$ \\ Nobuaki Shimizu, ${ }^{1}$ Kazuhiro Suzuki ${ }^{1}$ and Hidetoshi Yamanaka ${ }^{1}$
}

\begin{abstract}
Follow-up pelvic MRI incidentally revealed that a 35-year old female patient had an urachal cyst on the bladder dome. A histerectomy for cervical cancer had previously been performed. The urachal cyst was $1.1 \mathrm{~cm}$ in diameter and transurethral en bloc resection was successfully performed. Histopathologically, the cyst was surrounded by a smooth muscle layer with the wall consisting of columnar and glandular epithelial cells. Transurethral en bloc resection is a useful procedure to excise and confirm the pathogenesis for a limited case of a small urachal cyst that extrudes into the urinary bladder. (Kitakanto Med J $2003 ; 53: 323 \sim 324)$
\end{abstract}

Key words : urachal cyst, transurethral resection

\section{Introduction}

The presence of an urachal cyst may sometimes be revealed by the investigation of an infectious condition and may lead to the development of an urachal tumor. ${ }^{1}$ Small cyst remains asymptomatic and is incidentally detected by imaging. Therapeutic options widely varied, but partial cystectomy is essential for an urachal cyst on the bladder dome. ${ }^{1 \sim 3}$ We demonstrate endoscopic en bloc resection of a non-infectious urachal cyst.

\section{Case Report}

Pelvic MRI imaging incidentally revealed a small rounded tumor on the bladder dome of a 35-year-old female patient in November 2001. The patient had a past history of cervical cancer and was still being observed during following period of five years. Cystoscopy revealed an urachal cyst $1.1 \mathrm{~cm}$ in diameter on top of the bladder dome. Urinalysis was normal and urine cytology was class I. Though there were no signs of inflammation and infection, malignancy of the urachus could not be disregarded. In February 2002, transurethral en bloc resection of the urachal cyst was successfully performed under spinal anesthesia (Fig. 1). The border of resection was marked by coagulating mode and resected the surface by cutting mode with a knife-type electrode. In subepiththelial area, a section of superficial muscle layer of the bladder was carefully observed and cut to remove the cyst. The cyst was held between a loop electrode and tip of the sheath and removed out of the urethra. The operative time was 9 minutes, the amount of bleeding was a little and the border of the resection was limited to the muscular bladder wall with no extravesical perforation. The post operative course was unremarkable. An indwelling catheter was transurethrally placed for two nights and the patient was discharged on post-operative day 2. Histopathologically, the urachal cyst was surrounded by a smooth muscle layer with the internal wall consisted of columnar and secretory glandular epithelial cells (Fig. 2). There is no evidence of the recurrences in both uterus cancer and urachal cyst in December 2002.

\section{Discussion}

The urachus lies between the peritoneum and the transversalis fascia and extends from the anterior dome of the bladder to the umbilicus. An urachal cyst may form within the isolated canal with the locations of urachal cysts varying widely. ${ }^{1}$ A connection persists between the tract and the bladder and may cause bacterial infection. However, the cyst that protrudes into the bladder without connecting with the umbilical tract, rarely exhibits this infectious feature and remains asymptomatic. ${ }^{1,4}$ This type of urachal cyst may be incidentally found by imaging, such as ultrasonography, CT and MRI.

1 Department of Urology, Gunma University Graduate School of Medicine, Maebashi, Japan

Received : July 4, 2003

Address : KAZUHIRO SUZUKI Department of Urology, Gunma University Graduate School of Medicine, 3-39-22 Showa-machi, Maebashi, Gunma 371-8511, Japan 

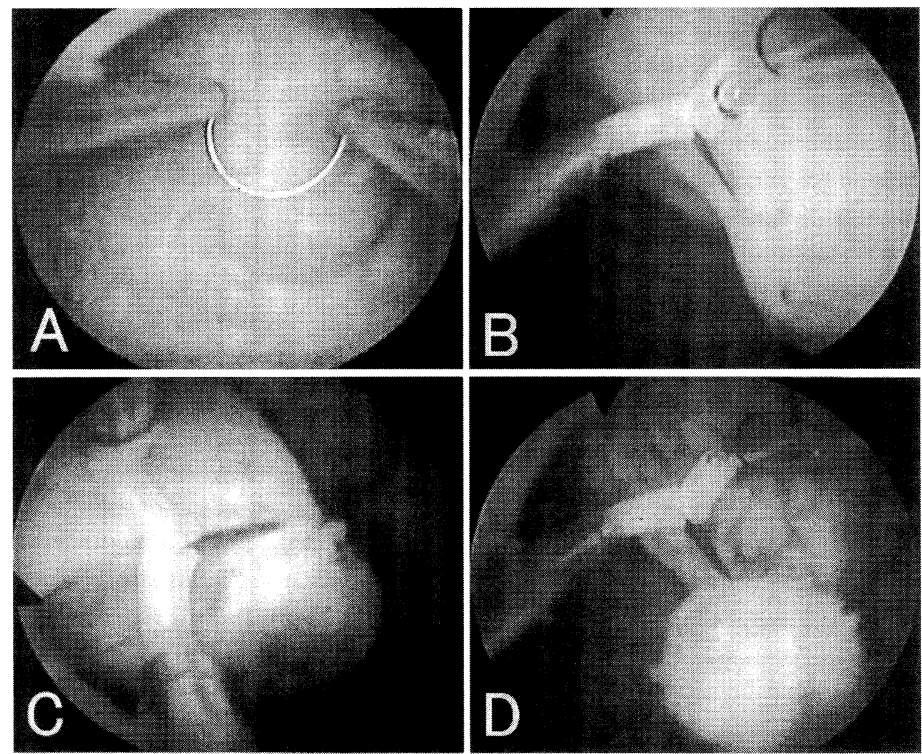

Fig. 1. Endoscopic features of the transurethral en bloc resection of an urachal cyst.

A : The surface of the cyst is smooth and $1.1 \mathrm{~cm}$ in diameter (resection loop: $0.6 \mathrm{~cm}$ in diameter).

$\mathrm{B}, \mathrm{C}$ : The bladder mucosa was carefully incised around the cyst to prevent of the rupture.

D : Muscle fibers were hooked and incised. Subsequently, the cyst was removed and no connection with the extravesical area was made.

Partial cystectomy is essential for the treatment of an urachal cyst on the bladder dome. Recently, laparoscopic excisions of entire cysts have been performed with demonstration of the minimally invasive and safe techniques involved. ${ }^{2,3}$ However, transurethral en bloc resection had never been performed with an urachal cyst. Transurethral en bloc resection is performed for the treatment of the sessile bladder tumor and the resection electrodes have been developed. ${ }^{5}$ We considered the following three conditions: First, the urachal cyst must extrude into the bladder. Second, the cyst must not be connected to the upper urachal duct or have any other urachal abnormality. This allows completion of the procedure with little risk of perforating or making a large defect in the bladder wall. Third, the size must be small enough to be transurethrally removed. This allows removal of the cyst without bursting the cyst wall and disseminating its internal material.

In this report, a patient who underwent the relatively uncommon transurethral en bloc resection was presented. This procedure is minor invasive than those of partial cystectomy and laparoscopic procedure, but it is adapted for a limited case of a small urachal cyst that extrudes into the urinary bladder like this reported case.

\section{References}

1. Gearhart JP, Jeffs RD. Extrophy-epispadias complex and bladder anomalies. In : Walsh PC, Retik AB, Vaughan ED (eds). Campbell's urology, 7th ed. Philadelphia: WB Saunders, 1998:

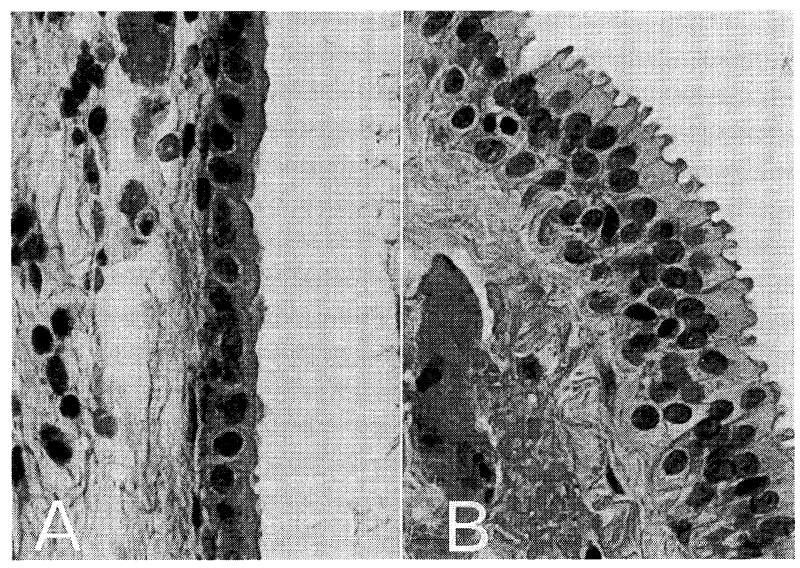

Fig. 2. A : The cyst wall consisted of a single layer of columnar epithelial cells.

B : The cyst wall may also contain excretory glandular epithelial cells. No atypical cell or no malignant features were seen. (Hematoxilin and Eosin staining, $\times 400$ )

2497-2505.

2. Siegel JF, Winfield HN, Valderrama E, et al. Laparoscopic excision of urachal cyst. J Urol $1994 ; 151: 1631-1633$.

3. Stone NN, Garden RJ, Weber H. Laparoscopic excision of an urachal cyst. Urology $1995 ; 45$ : 161-164.

4. Berman SM, Reid RE, Tolia BM, et al. Urachal remnants in adults. Urology 1988; 31: 17-21.

5. Kawada T, Ebihara K, Suzuki T, et al. A new technique for transurethral resection of bladder tumors : rotational tumor resection using a new arched electrode. J. Urol 1997; 157 : 22252226. 